TRANSACTIONS OF THE

AMERICAN MATHEMATICAL SOCIETY

Volume 366, Number 1, January 2014, Pages 531-546

S 0002-9947(2013)05895-3

Article electronically published on June 18, 2013

\title{
PIERI OPERATORS ON THE AFFINE NILCOXETER ALGEBRA
}

\author{
CHRIS BERG, FRANCO SALIOLA, AND LUIS SERRANO
}

\begin{abstract}
We study a family of operators on the affine nilCoxeter algebra. We use these operators to prove conjectures of Lam, Lapointe, Morse, and Shimozono regarding strong Schur functions.
\end{abstract}

\section{INTRODUCTION}

The $k$-Schur functions of Lapointe, Lascoux and Morse [LLM03 first arose in the study of Macdonald polynomials. Since then, their study has flourished; see for instance LM03, LM05,LM07,LS07,LLMS10,Lam10 and the references therein. This is due, in part, to an important geometric interpretation of the Hopf algebra $\Lambda_{(k)}$ of $k$-Schur functions and its dual Hopf algebra $\Lambda^{(k)}$ : these algebras are isomorphic to the homology and cohomology of the affine Grassmannian in type A [Lam08]. Under this isomorphism, the $k$-Schur functions map to the Schubert basis of the homology and the dual $k$-Schur functions (also called the affine Schur functions) map to the Schubert basis of the cohomology.

An important problem in the theory of $k$-Schur functions is to find a $k$-Littlewood-Richardson rule, namely, a combinatorial interpretation for the (nonnegative) coefficients in the expansion

$$
s_{\mu}^{(k)} s_{\nu}^{(k)}=\sum_{\lambda} c_{\mu, \nu}^{\lambda,(k)} s_{\lambda}^{(k)} .
$$

The $c_{\mu, \nu}^{\lambda,(k)}$ are called the $k$-Littlewood-Richardson coefficients, and are of high relevance in combinatorics and geometry. It was proved by Lapointe and Morse LM08] that special cases of these coefficients yield the 3-point Gromov-Witten invariants. These invariants are the structure constants of the quantum cohomology of the Grassmanian; they count the number of rational curves of a fixed degree in the Grassmannian. The 3-point Gromov-Witten invariants of flag varieties are also $k$-Littlewood-Richardson coefficients; see LS10,LL12.

As an approach to finding the $k$-Littlewood-Richardson coefficients, Lam Lam06 identified $\Lambda_{(k)}$ with the affine Fomin-Stanley subalgebra $\mathbb{B}$ of the affine nilCoxeter algebra $\mathbb{A}$ of the affine symmetric group $W$. Specifically, he constructed a family of elements $\mathfrak{s}_{\lambda}^{(k)} \in \mathbb{B}$ that map under this isomorphism to the $k$-Schur functions $s_{\lambda}^{(k)}$. Furthermore, he proved Lam06, Proposition 42] that finding the $k$-Littlewood-Richardson rule is equivalent to finding the expansion of $\mathfrak{s}_{\lambda}^{(k)}$ in the "standard basis" $\mathbf{u}_{w}$ of $\mathbb{A}$. Explicitly, he proved that the coefficients in (1) appear

Received by the editors March 29, 2012 and, in revised form, June 12, 2012.

2010 Mathematics Subject Classification. Primary 05E05; Secondary 14N15.

(c) 2013 American Mathematical Society 
as coefficients in the expansion

$$
\mathfrak{s}_{\lambda}^{(k)}=\sum_{w \in W} d_{\lambda}^{w} \mathbf{u}_{w}
$$

In this article, we develop a family of operators on $\mathbb{A}$, which will facilitate the study of $\mathfrak{s}_{\lambda}^{(k)}$, and we prove certain conjectures regarding a family of functions that generalize the $k$-Schur functions $s_{\lambda}^{(k)}$. Each of these is described in more detail below.

1.1. The Pieri operators. Lam, Lapointe, Morse, and Shimozono [LLMS10] constructed a labelled directed graph $\mathcal{G}^{\downarrow}$ on the elements of $W$, which encompasses the strong order in $W$. In this article we study the operators on $\mathbb{A}$ induced by the Pieri operators of $\mathcal{G}^{\downarrow}$ in the spirit of [BMSvW00]. In Section 4, we develop the main properties of these operators. More specifically, in Theorem 4.9 we prove that these operators are determined by their restriction to $\mathbb{B}$, in Theorem 4.7 we determine this restriction, and in Theorem 4.10 we prove that the operators commute pairwise.

1.2. Properties of strong Schur functions. Lam, Lapointe, Morse, and Shimozono LLMS10] generalized $s_{\lambda}^{(k)}$ to a larger set of functions called the strong Schur functions $\operatorname{Strong}_{u / v}$, where $u$ and $v$ are any pair of elements in $W$. In Section 5, we use the Pieri operators to prove a series of conjectures of Lam, Lapointe, Morse, and Shimozono [LLMS10, Conjecture 4.18] regarding these functions. Specifically,

(a) in Theorem 5.2 we prove that Strong ${ }_{u / v}$ are symmetric functions;

(b) in Theorem 5.4 we prove that they belong to the algebra $\Lambda_{(k)}$; and

(c) in Theorem 5.5 we describe the coefficient of $s_{\lambda}^{(k)}$ in Strong ${ }_{u / v}$, when $u$ and $v$ are 0-Grassmannian elements, in terms of the structure constants of the cohomology ring of the affine flag variety.

Note that (c) provides a combinatorial description of the skew $k$-Schur functions.

\section{BACKGROUND AND NOTATION}

2.1. Affine symmetric group. Fix a positive integer $k$. Let $W$ denote the affine symmetric group with simple generators $s_{0}, s_{1}, \ldots, s_{k}$. There is an interpretation of $W$ as the group of permutations $w: \mathbb{Z} \rightarrow \mathbb{Z}$ satisfying $w(i+k+1)=w(i)+k+1$ for all $i \in \mathbb{Z}$ and $\sum_{i=1}^{k+1} w(i)=\sum_{i=1}^{k+1} i$. For two integers $i$ and $j$ that are not congruent modulo $k+1$, let $t_{i, j}$ be the element of $W$ that interchanges $i$ and $j$ and fixes all integers not congruent to $i$ or $j$ modulo $k+1$.

Let $W_{0}$ denote the subgroup of $W$ generated by $s_{1}, \ldots, s_{k}$ and let $W^{0}$ denote the set of minimal length coset representatives of $W / W_{0}$. Elements of $W^{0}$ are called affine Grassmannian elements or 0-Grassmannian elements. There are bijections between 0 -Grassmannian elements, $k$-bounded partitions, and $(k+1)$-cores. We will not review these here, but refer the reader to [LM05]. For a $k$-bounded partition $\lambda$, we let $w_{\lambda}$ denote the corresponding element of $W^{0}$. Let $\mathcal{B}^{(k)}$ denote the set of $k$-bounded partitions. 
2.2. Affine nilCoxeter algebra. Let $\mathbb{A}$ denote the affine nilCoxeter algebra of $W$. This is the algebra generated by $\mathbf{u}_{0}, \mathbf{u}_{1}, \ldots, \mathbf{u}_{k}$ with relations

$$
\begin{gathered}
\mathbf{u}_{i}^{2}=0 \text { for all } i \\
\mathbf{u}_{i} \mathbf{u}_{i+1} \mathbf{u}_{i}=\mathbf{u}_{i+1} \mathbf{u}_{i} \mathbf{u}_{i+1} \text { with } i+1 \text { taken modulo } k+1, \\
\mathbf{u}_{i} \mathbf{u}_{j}=\mathbf{u}_{j} \mathbf{u}_{i} \text { if } i-j \neq \pm 1 \text { modulo } k+1
\end{gathered}
$$

It follows that a basis of $\mathbb{A}$ is given by the elements $\mathbf{u}_{w}=\mathbf{u}_{s_{i_{1}}} \mathbf{u}_{s_{i_{2}}} \cdots \mathbf{u}_{s_{i_{l}}}$, where $w=s_{i_{1}} s_{i_{2}} \cdots s_{i_{l}}$ is a reduced word for $w \in W$. We define an inner product on $\mathbb{A}$ by $\left\langle\mathbf{u}_{v}, \mathbf{u}_{w}\right\rangle_{\mathbb{A}}=\delta_{u, v}$.

2.3. Affine Fomin-Stanley subalgebra. An element $w \in W$ is said to be cyclically decreasing if there exists a reduced factorization $s_{i_{1}} \cdots s_{i_{j}}$ of $w$ satisfying: each letter occurs at most once; and, for all $m$, if $s_{m}$ and $s_{m+1}$ both appear in the reduced factorization, then $s_{m+1}$ precedes $s_{m}$. If $D \subsetneq\{0,1, \ldots, k\}$, then there is a unique cyclically decreasing element $w_{D}$ with letters $\left\{s_{d}: d \in D\right\}$. Let $\mathbf{u}_{D}=\mathbf{u}_{w_{D}}$ denote the corresponding basis element of $\mathbb{A}$. For $i \in\{0,1, \ldots, k\}$, let

$$
\mathbf{h}_{i}=\sum_{\substack{D \subset I \\|D|=i}} \mathbf{u}_{D} \in \mathbb{A} .
$$

By a result of Thomas Lam [Lam06], the elements $\left\{\mathbf{h}_{i}\right\}_{i \leq k}$ commute and freely generate a subalgebra $\mathbb{B}$ of $\mathbb{A}$ called the affine Fomin-Stanley subalgebra. The elements $\mathbf{h}_{\lambda}=\mathbf{h}_{\lambda_{1}} \ldots \mathbf{h}_{\lambda_{t}}$, for all $k$-bounded partitions $\lambda=\left(\lambda_{1}, \ldots, \lambda_{t}\right)$, form a basis of $\mathbb{B}$.

2.4. Symmetric functions. Let $\Lambda$ denote the ring of symmetric functions. For a partition $\lambda$, we let $m_{\lambda}, h_{\lambda}, e_{\lambda}, p_{\lambda}, s_{\lambda}$ denote the monomial, homogeneous, elementary, power sum and Schur symmetric functions, respectively, indexed by $\lambda$. Each of these families forms a basis of $\Lambda$. We recall the following change of bases formulae:

$$
h_{\mu}=\sum_{\lambda} K_{\lambda, \mu} s_{\lambda} \quad \text { and } \quad s_{\lambda}=\sum_{\mu} K_{\lambda, \mu} m_{\mu}
$$

where $K_{\lambda, \mu}$, called the Kostka number, is the number of semistandard tableaux of shape $\lambda$ and content $\mu$ Sta.

Let $\Lambda_{(k)}$ denote the subalgebra of $\Lambda$ generated by $h_{0}, h_{1}, \ldots, h_{k}$. The elements $h_{\lambda}$ with $\lambda_{1} \leq k$ form a basis of $\Lambda_{(k)}$. Let $\Lambda^{(k)}=\Lambda / I_{k}$ denote the quotient of $\Lambda$ by the ideal $I_{k}$ generated by $m_{\lambda}$ with $\lambda_{1}>k$. The equivalence classes in $\Lambda^{(k)}$ of the elements $m_{\lambda}$ with $\lambda_{1} \leq k$ form a basis of $\Lambda^{(k)}$.

The Hall inner product of symmetric functions is defined by

$$
\left\langle h_{\lambda}, m_{\mu}\right\rangle_{\Lambda}=\left\langle s_{\lambda}, s_{\mu}\right\rangle_{\Lambda}=\delta_{\lambda, \mu} .
$$

Observe that every element of the ideal $I_{k}$ is orthogonal to every element of $\Lambda_{(k)}$ with respect to this inner product. Hence, it induces a pairing $\langle\cdot, \cdot\rangle$ between $\Lambda_{(k)}$ and $\Lambda^{(k)}$. In particular, $\langle f, g\rangle=\langle f, \widetilde{g}\rangle$ for $f \in \Lambda_{(k)}, g \in \Lambda^{(k)}$ and any preimage $\widetilde{g}$ of $g$ under the quotient map $\Lambda \rightarrow \Lambda^{(k)}$. For an element $f$ in $\Lambda^{(k)}$, write $f^{\perp}: \Lambda_{(k)} \rightarrow \Lambda_{(k)}$ for the linear operator that is adjoint to multiplication by $f$ with respect to $\langle\cdot, \cdot\rangle$. 
2.5. Affine Schur functions. The affine Schur functions form a distinguished basis of $\Lambda^{(k)}$. For $w \in W$, the affine Stanley symmetric function is defined as

$$
\widetilde{F}_{w}=\sum_{\lambda \in \mathcal{B}(k)}\left\langle\mathbf{h}_{\lambda}, \mathbf{u}_{w}\right\rangle_{\mathbb{A}} m_{\lambda},
$$

where $m_{\lambda}$ is the monomial symmetric function indexed by $\lambda$. These functions are elements of $\Lambda^{(k)}$, but they are not linearly independent. For a $k$-bounded partition $\lambda$, let $\widetilde{F}_{\lambda}=\widetilde{F}_{w_{\lambda}}$, where $w_{\lambda}$ denotes the 0-Grassmannian element corresponding to $\lambda$. The functions $\widetilde{F}_{\lambda}$ are called affine Schur functions (or dual $k$-Schur functions), and they form a basis of $\Lambda^{(k)}$. See for instance [Lam06, LM08].

2.6. $k$-Schur functions. The $k$-Schur functions are a distinguished basis of $\Lambda_{(k)}$. They are defined as the duals of the affine Schur functions with respect to the inner product $\langle\cdot, \cdot\rangle$ on $\Lambda_{(k)} \times \Lambda^{(k)}$. That is, they satisfy $\left\langle s_{\lambda}^{(k)}, \widetilde{F}_{\mu}\right\rangle=\delta_{\lambda, \mu}$ for all $k$-bounded partitions $\lambda$ and $\mu$. Equivalently, they are uniquely defined by the $k$-Pieri rule:

$$
h_{i} s_{\lambda}^{(k)}=\sum s_{\nu}^{(k)},
$$

where the sum ranges over all $k$-bounded partitions $\nu$ such that $w_{\nu} w_{\lambda}^{-1}$ is cyclically decreasing of length $i$. It follows from duality and (3) that

$$
h_{\mu}=\sum_{\lambda \in \mathcal{B}^{(k)}}\left\langle\mathbf{h}_{\lambda}, \mathbf{u}_{w_{\mu}}\right\rangle_{\mathbb{A}} s_{\lambda}^{(k)}
$$

2.7. Noncommutative $k$-Schur functions. The algebras $\Lambda_{(k)}$ and $\mathbb{B}$ are isomorphic with isomorphism given by $h_{\lambda} \mapsto \mathbf{h}_{\lambda}$. We denote by $\mathfrak{s}_{\lambda}^{(k)}$ the image of the $k$-Schur function $s_{\lambda}^{(k)}$ under this isomorphism. In the literature, $\mathfrak{s}_{\lambda}^{(k)}$ is called a noncommutative $k$-Schur function. They have the following expansion Lam06, Proposition 42]:

$$
\mathfrak{s}_{\lambda}^{(k)}=\sum_{w \in W}\left\langle s_{\lambda}^{(k)}, \widetilde{F}_{w}\right\rangle \mathbf{u}_{w}
$$

That is, the coefficient of $\mathbf{u}_{w}$ in $\mathfrak{s}_{\lambda}^{(k)}$ is equal to the coefficient of $\widetilde{F}_{\lambda}$ in $\widetilde{F}_{w}$ :

$$
\left\langle s_{\lambda}^{(k)}, \widetilde{F}_{w}\right\rangle=\left\langle\mathfrak{s}_{\lambda}^{(k)}, \mathbf{u}_{w}\right\rangle_{\mathbb{A}},
$$

and so

$$
\widetilde{F}_{w}=\sum_{\lambda \in \mathcal{B}^{(k)}}\left\langle\mathfrak{s}_{\lambda}^{(k)}, \mathbf{u}_{w}\right\rangle_{\mathbb{A}} \widetilde{F}_{\lambda}
$$

Consequently, $\mathfrak{s}_{\lambda}^{(k)}$ contains exactly one term $\mathbf{u}_{w}$ with $w \in W^{0}$ and its coefficient is 1. Furthermore, if $\sum_{w} c_{w} \mathbf{u}_{w}$ is known to lie in $\mathbb{B}$, then $\sum_{w} c_{w} \mathbf{u}_{w}=\sum_{\lambda} c_{w_{\lambda}} \mathfrak{s}_{\lambda}^{(k)}$.

\section{DeFinition OF THE OPERATORS}

In this section, we define operators on the affine nilCoxeter algebra $\mathbb{A}$. The definitions are dependent upon the combinatorics introduced by Lam, Lapointe, Morse, and Shimozono in [LLMS10. 


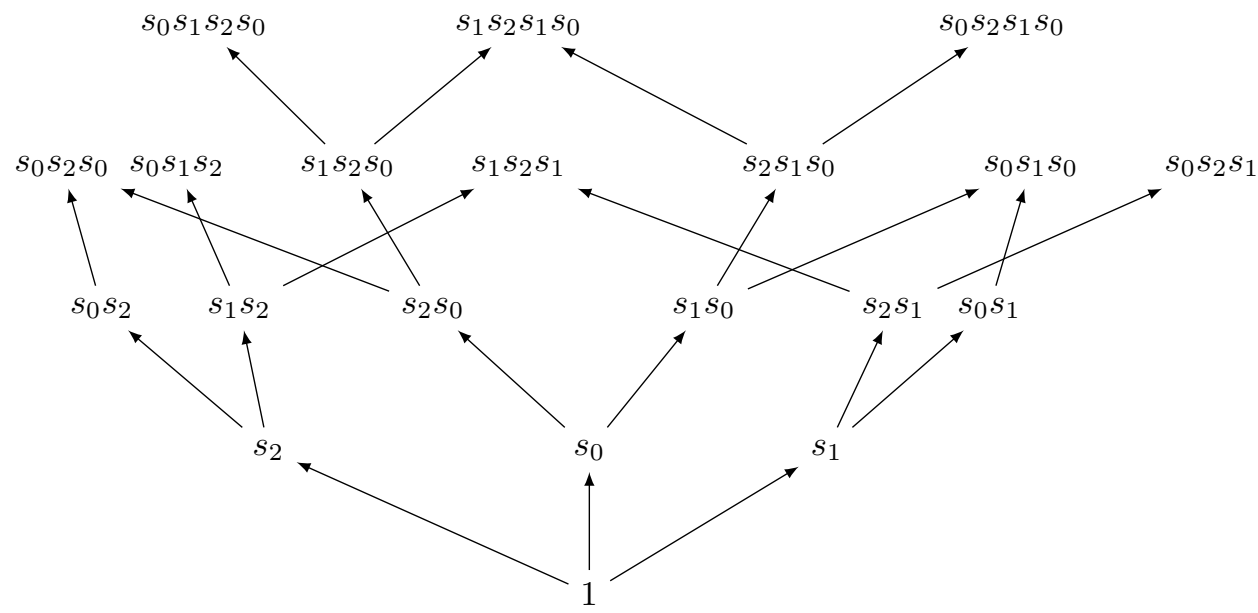

FiguRE 1. A subgraph of $\mathcal{G}^{\uparrow}$ for $k=2$; cf. Figure 2 .

3.1. Up operators. Define an edge-labelled oriented graph $\mathcal{G}^{\uparrow}$ with vertex set $W$ : there is an edge from $v$ to $w$ labelled by $i$ whenever $\ell(w)=\ell(v)+1$ and $s_{i} v=w$. (See Figure 1) So, $\mathcal{G}^{\uparrow}$ is the graph for the (left) weak order on $W$.

A weak strip of length $j$ from $w$ to $v$, denoted by $w \rightsquigarrow v$, is a pair of elements $w, v \in W$ such that $w$ precedes $v$ in weak order and $v w^{-1}$ is a cyclically decreasing word of length $j$.

For any nonnegative integer $j$, define a linear operator $U_{j}: \mathbb{A} \rightarrow \mathbb{A}$ by

$$
U_{j}\left(\mathbf{u}_{w}\right)=\sum_{\substack{w \rightsquigarrow v \\ \operatorname{size}(w \rightsquigarrow v)=j}} \mathbf{u}_{v}=\mathbf{h}_{j} \mathbf{u}_{w},
$$

where the sum ranges over all weak strips of length $j$ that begin at $w$. Equivalently, $U_{j}$ is multiplication on the left by $\mathbf{h}_{j}$.

Example 3.1. With $k=2$ : $U_{1}\left(\mathbf{u}_{0}\right)=\mathbf{u}_{2} \mathbf{u}_{0}+\mathbf{u}_{1} \mathbf{u}_{0}$ and $U_{2}\left(\mathbf{u}_{0}\right)=\mathbf{u}_{0} \mathbf{u}_{2} \mathbf{u}_{0}+\mathbf{u}_{2} \mathbf{u}_{1} \mathbf{u}_{0}$.

3.2. Down operators. Define a second edge-labelled oriented graph $\mathcal{G}^{\downarrow}$, the marked strong order graph, with vertex set $W$ : there is an edge from $x$ to $y$ labelled by $y(j)=x(i)$ whenever $\ell(x)=\ell(y)+1$ and there exists $i \leq 0<j$ such that $y t_{i, j}=x$.

Example $3.2(k=2)$. There are two edges from $x=s_{0} s_{1} s_{2} s_{0}$ to $y=s_{1} s_{2} s_{0}$ since $y^{-1} x$ can be written as $t_{i, j}$ with $i \leq 0<j$ in two ways: $y^{-1} x=t_{-4,1}=t_{-1,4}$. These edges are labelled by $y(1)=-2$ and $y(4)=1$. See Figure 2

Remark 3.3. The authors in [LLMS10] defined a similar graph except that they oriented their edges in the opposite direction and labelled the edges by the pair $(i, j)$ : they wrote $y \stackrel{i, j}{\longrightarrow} x$ whereas we write $x \stackrel{y(j)}{\longrightarrow} y$; and they called our label $y(j)$ the marking of the edge.

A strong strip of length $i$ from $w$ to $v$, denoted by $w \rightarrow v$, is a path

$$
w \stackrel{\ell_{1}}{\longrightarrow} w_{1} \stackrel{\ell_{2}}{\longrightarrow} \cdots \stackrel{\ell_{i}}{\longrightarrow} w_{i}=v
$$




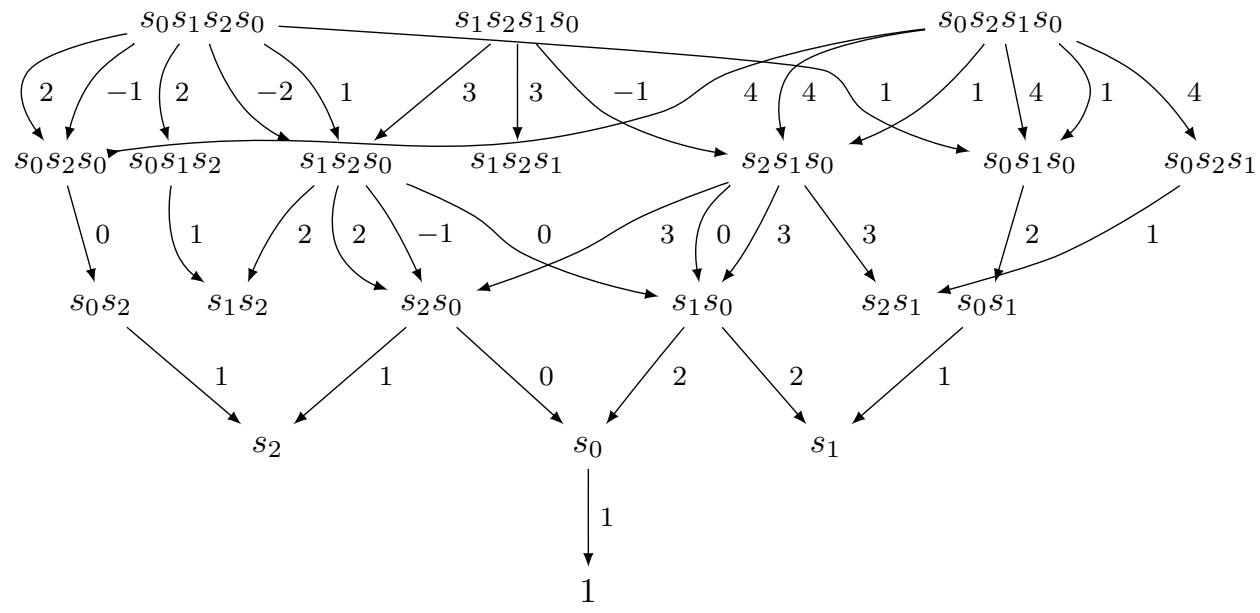

FiguRE 2. $\mathcal{G}^{\downarrow}$ for $k=2$ truncated at the affine Grassmannian elements of length 4.

of length $i$ in $\mathcal{G}^{\downarrow}$ with decreasing edge labels: $\ell_{1}>\cdots>\ell_{i}$. For nonnegative integers $i$, define $D_{i}: \mathbb{A} \rightarrow \mathbb{A}$ as

$$
D_{i}\left(\mathbf{u}_{w}\right)=\sum_{\substack{w \rightarrow v \\ \operatorname{size}(w \rightarrow v)=i}} \mathbf{u}_{v},
$$

where the sum ranges over all strong strips of length $i$ that begin at $w$. In particular, the coefficient of $\mathbf{u}_{v}$ in $D_{i}\left(\mathbf{u}_{w}\right)$ is the number of strong strips of length $i$ that begin at $w$ and end at $v$.

Example 3.4. With $k=2$, using the graph from Figure 2, one can verify that

$$
\begin{aligned}
& D_{1}\left(\mathbf{u}_{0} \mathbf{u}_{1} \mathbf{u}_{2} \mathbf{u}_{0}\right)=2 \mathbf{u}_{0} \mathbf{u}_{2} \mathbf{u}_{0}+\mathbf{u}_{0} \mathbf{u}_{1} \mathbf{u}_{2}+2 \mathbf{u}_{1} \mathbf{u}_{2} \mathbf{u}_{0}+\mathbf{u}_{0} \mathbf{u}_{1} \mathbf{u}_{0}, \\
& D_{2}\left(\mathbf{u}_{0} \mathbf{u}_{1} \mathbf{u}_{2} \mathbf{u}_{0}\right)=\mathbf{u}_{0} \mathbf{u}_{2}+\mathbf{u}_{1} \mathbf{u}_{2}+\mathbf{u}_{2} \mathbf{u}_{0}+\mathbf{u}_{1} \mathbf{u}_{0} .
\end{aligned}
$$

More generally, we define an operator $D_{J}$ for any composition $J$ of positive integers; the operator $D_{i}$ defined above is $D_{J}$ for the composition $J=[i]$. We need some additional notation. The ascent composition of a sequence $\ell_{1}, \ell_{2}, \ldots, \ell_{m}$ is the composition $\left[i_{1}, i_{2}-i_{1}, \ldots, i_{j}-i_{j-1}, m-i_{j}\right]$, where $i_{1}<i_{2}<\cdots<i_{j}$ are the ascents of the sequence; that is, the elements in $\{1, \ldots, m-1\}$ such that $\ell_{i_{a}}<\ell_{i_{a}+1}$. For example, the ascent composition of the sequence $3,2,0,3,4,1$ is $[3,1,2]$ since the ascents are in positions 3 and 4 .

If $w_{0} \stackrel{\ell_{1}}{\longrightarrow} \cdots \stackrel{\ell_{m}}{\longrightarrow} w_{m}$ is a path in $\mathcal{G}^{\downarrow}$, then we let $\operatorname{ascomp}\left(w_{0} \stackrel{\ell_{1}}{\longrightarrow} \cdots \stackrel{\ell_{m}}{\longrightarrow}\right.$ $w_{m}$ ) denote the ascent composition of the sequence of labels $\ell_{1}, \ldots, \ell_{m}$. It is a composition of the length of the path.

For a composition $J=\left[j_{1}, j_{2}, \ldots, j_{l}\right]$ of positive integers, define

$$
D_{J}\left(\mathbf{u}_{w}\right)=\sum_{\operatorname{ascomp}\left(w \stackrel{\ell_{1}}{\longrightarrow} w_{1} \stackrel{\ell_{2}}{\longrightarrow} \ldots \stackrel{\ell_{m}}{\longrightarrow} w_{m}\right)=J} \mathbf{u}_{w_{m}},
$$

where the sum ranges over all paths in $\mathcal{G}^{\downarrow}$ of length $m=j_{1}+\cdots+j_{l}$ beginning at $w$ whose sequence of labels has ascent composition $J$. 
Example 3.5. With $k=2$ one can verify using Figure 2 that

$$
\begin{aligned}
D_{[3]}\left(\mathbf{u}_{1} \mathbf{u}_{2} \mathbf{u}_{1} \mathbf{u}_{0}\right) & =\mathbf{u}_{2}+\mathbf{u}_{0}, \\
D_{[2,1]}\left(\mathbf{u}_{1} \mathbf{u}_{2} \mathbf{u}_{1} \mathbf{u}_{0}\right) & =\mathbf{u}_{2}+2 \mathbf{u}_{0}+\mathbf{u}_{1}, \\
D_{[1,2]}\left(\mathbf{u}_{1} \mathbf{u}_{2} \mathbf{u}_{1} \mathbf{u}_{0}\right) & =\mathbf{u}_{2}+2 \mathbf{u}_{0}+\mathbf{u}_{1}, \\
D_{[1,1,1]}\left(\mathbf{u}_{1} \mathbf{u}_{2} \mathbf{u}_{1} \mathbf{u}_{0}\right) & =\mathbf{u}_{0}+\mathbf{u}_{1} .
\end{aligned}
$$

For two compositions $I=\left[i_{1}, \ldots, i_{r}\right]$ and $J=\left[j_{1}, \ldots, j_{s}\right]$, let

$$
\begin{aligned}
& I \boxplus J=\left[i_{1}, \ldots, i_{r-1}, i_{r}+j_{1},, j_{2}, \ldots, j_{s}\right], \\
& I \boxminus J=\left[i_{1}, \ldots, i_{r}, j_{1}, \ldots, j_{s}\right] .
\end{aligned}
$$

Proposition 3.6. If $I$ and $J$ are compositions, then

$$
D_{I} \circ D_{J}=D_{I \boxplus J}+D_{I \oplus J} .
$$

Proof. If $w \rightarrow \cdots \rightarrow v$ and $v \rightarrow \cdots \rightarrow u$ are two paths in $\mathcal{G}^{\downarrow}$ with ascent compositions $J$ and $I$, respectively, then the path $w \rightarrow \cdots \rightarrow v \rightarrow \cdots \rightarrow u$ has ascent composition either $I \ominus J$ or $I \boxplus J$.

Corollary 3.7. Suppose $I=\left[i_{1}, \ldots, i_{r}\right]$ is a composition. Then

$$
\sum_{J \preceq I} D_{J}=D_{i_{1}} \circ \cdots \circ D_{i_{r}}
$$

where $\preceq$ denotes reverse refinement order on compositions 1

Proof. Proceed by induction on $r$. This is trivially true for $r=1$. Suppose the result holds for compositions of length less than $r$. Then

$$
D_{i_{1}} \circ \cdots \circ D_{i_{r}}=D_{i_{1}}\left(\sum_{J^{\prime} \preceq\left[i_{2}, \ldots, i_{r}\right]} D_{J^{\prime}}\right)=\sum_{J^{\prime} \preceq\left[i_{2}, \ldots, i_{r}\right]}\left(D_{\left[i_{1}, j_{1}, \ldots, j_{s}\right]}+D_{\left[i_{1}+j_{1}, \ldots, j_{s}\right]}\right),
$$

which is $\sum_{J \preceq I} D_{J}$ since the first part of a composition $J$ that satisfies $J \preceq I$ is either $i_{1}$ or $i_{1}+\left(i_{2}+\cdots+i_{l}\right)$ for some $l \geq 2$.

\section{Properites of the operators}

In this section we develop properties of the operators $U_{j}$ and $D_{i}$.

4.1. Extensions of linear endomorphisms of $\mathbb{B}$ to $\mathbb{A}$. Since $W^{0}$ is a set of coset representatives of $W_{0}$ in $W$, every element $w$ of $W$ factors uniquely as $w=w^{(0)} w_{(0)}$ with $w^{(0)} \in W^{0}$ and $w_{(0)} \in W_{0}$. We call this the 0-Grassmannian factorization of $w$. Since the elements of $W^{0}$ are in bijection with $k$-bounded partitions, we can write this factorization as $w=w_{\lambda} w_{(0)}$, and we let

$$
\mathbf{b}_{w}=\mathfrak{s}_{\lambda}^{(k)} \mathbf{u}_{w_{(0)}} .
$$

Proposition 4.1. The set $\left\{\mathbf{b}_{w}: w \in W\right\}$ is a basis of $\mathbb{A}$.

\footnotetext{
${ }^{1}$ With respect to this order, the composition $[1,1, \ldots, 1]$ is the maximal element and the composition $[n]$ is the minimal element.
} 
Proof. We will define a total order on the elements of $W$ in such a way that the leading term of $\mathbf{b}_{w}$ is $\mathbf{u}_{w}$. Then, with respect to this ordering, the transition matrix from $\left\{\mathbf{b}_{w}\right\}$ to $\left\{\mathbf{u}_{w}\right\}$ is uni-triangular, from which the result follows.

Informally, we need an order in which $v$ precedes $u$ whenever $\ell(u)>\ell(v)$ or the "Grassmannian part" of $u$ is bigger than that of $v$. Define $v$ to precede $u$ if: $\ell(u)>\ell(v)$; or $\ell(u)=\ell(v)$ and $\ell\left(u^{(0)}\right)>\ell\left(v^{(0)}\right)$. Note that this is only a partial order, but any linear extension of this partial order will do the trick.

First we argue that the leading term of $\mathbf{b}_{w_{\lambda}}=\mathfrak{s}_{\lambda}^{(k)}$ is $\mathbf{u}_{w_{\lambda}}$. Indeed, $\mathfrak{s}_{\lambda}^{(k)}$ expanded in the basis $\left\{\mathbf{u}_{v}\right\}$ is a linear combination of terms $\mathbf{u}_{v}$ with the $v$ all of the same length $|\lambda|$, and it contains exactly one term $\mathbf{u}_{w}$ with $w \in W^{0}$, namely $w_{\lambda}$ (see 2.7).

Next, we prove that the leading term of $\mathbf{b}_{w}$ is $\mathbf{u}_{w}$. If $\mathbf{u}_{v}$ appears in $\mathbf{b}_{w}=\mathfrak{s}_{\lambda}^{(k)} \mathbf{u}_{w_{(0)}}$ with nonzero coefficient, then $\mathbf{u}_{v}=\mathbf{u}_{\tilde{v}} \mathbf{u}_{w_{(0)}}$ with $\mathbf{u}_{\tilde{v}}$ appearing in $\mathfrak{s}_{\lambda}^{(k)}$. It follows that $v=\tilde{v} w_{(0)}$ with $\ell(v)=\ell(\tilde{v})+\ell\left(w_{(0)}\right)$ and that $v^{(0)}=\tilde{v}^{(0)}$. Hence, to compare the terms $\mathbf{u}_{v}$ and $\mathbf{u}_{u}$ of $\mathbf{b}_{w}$, it suffices to compare the corresponding terms $\mathbf{u}_{\tilde{v}}$ and $\mathbf{u}_{\tilde{u}}$ of $\mathfrak{s}_{\lambda}^{(k)}$. So, the leading term of $\mathbf{b}_{w}$ is the leading term of $\mathfrak{s}_{\lambda}^{(k)}$ times $\mathbf{u}_{w_{(0)}}$, which is precisely $\mathbf{u}_{w_{\lambda}} \mathbf{u}_{w_{(0)}}=\mathbf{u}_{w}$.

Corollary 4.2. The set $\left\{\mathbf{h}_{\lambda} \mathbf{u}_{w_{(0)}}: w=w_{\lambda} w_{(0)} \in W\right\}$ is a basis of $\mathbb{A}$.

Proof. Follows from Proposition 4.1 and the fact that $\left\{\mathbf{h}_{\lambda}\right\}$ is a basis of $\mathbb{B}$.

The above results allow us to extend linear endomorphisms of $\mathbb{B}$ to linear endomorphisms of $\mathbb{A}$. Let $f: \mathbb{B} \rightarrow \mathbb{B}$ be a linear transformation of $\mathbb{B}$. Then we get a linear transformation $\widehat{f}: \mathbb{A} \rightarrow \mathbb{A}$ by defining $\widehat{f}$ on the basis $\left\{\mathbf{b}_{w}\right\}$ by

$$
\widehat{f}\left(\mathbf{b}_{w}\right)=\widehat{f}\left(\mathfrak{s}_{\lambda}^{(k)} \mathbf{u}_{w_{(0)}}\right)=f\left(\mathfrak{s}_{\lambda}^{(k)}\right) \mathbf{u}_{w_{(0)}},
$$

where $w=w_{\lambda} w_{(0)}$ is the 0 -Grassmannian factorization of $w$.

4.2. Commutation relation. We prove a commutation relation between the operators $U_{j}$ and $D_{i}$. This relation will allow us to bootstrap properties of $D_{1}$ and $U_{j}$ to every operator $D_{i}$ via an inductive argument.

Proposition 4.3 (Commutation relation).

$$
D_{i} \circ U_{j}=\sum_{e \geq 0} U_{j-e} \circ D_{i-e}
$$

Proof. First note that the right-hand side is a finite sum. The coefficient of $\mathbf{u}_{v}$ in

$$
\left(D_{i} \circ U_{j}\right)\left(\mathbf{u}_{u}\right)=\sum_{\substack{u \rightsquigarrow w \\ \operatorname{size}(u \rightsquigarrow w)=j}} \sum_{\substack{w \rightarrow v \\ \operatorname{size}(w \rightarrow v)=i}} \mathbf{u}_{v}
$$

is the number of tuples $(u \rightsquigarrow w, w \rightarrow v)$ consisting of a weak strip $u \rightsquigarrow w$ of length $j$ and a strong strip $w \rightarrow v$ of length $i$. The coefficient of $\mathbf{u}_{v}$ in

$$
\left(\sum_{e \geq 0} U_{j-e} \circ D_{i-e}\right)\left(\mathbf{u}_{u}\right)=\sum_{e \geq 0} \sum_{\substack{u \rightarrow x \\ \operatorname{size}(u \rightarrow x)=i-e}} \sum_{\substack{x \sim v \\ \operatorname{size}(x \rightsquigarrow v)=j-e}} \mathbf{u}_{v}
$$

is the number of triples $(e, u \rightarrow x, x \rightsquigarrow v)$ consisting of a nonnegative integer $e$, a strong strip $u \rightarrow x$ of length $i-e$ and a weak strip $x \rightsquigarrow v$ of length $j-e$. 
By [LLMS10, Proposition 4.1], these two numbers are the same. Indeed, that proposition establishes a bijection between the sets:

$$
\left\{\left(W^{\prime}, S^{\prime}\right): \begin{array}{l}
W^{\prime} \text { is a weak strip beginning at } u, \\
S^{\prime} \text { is a strong strip ending at } v, \\
\text { with } W^{\prime} \text { ending where } S^{\prime} \text { begins. }
\end{array}\right\}
$$

$$
\longleftrightarrow\left\{(W, S, e): \begin{array}{l}
W \text { is a weak strip ending at } v, \\
S \text { is a strong strip beginning at } u, \\
e \geq 0 \text { satisfies size }(W)+e \leq k, \\
\text { with } S \text { ending where } W \text { begins. }
\end{array}\right\}
$$

such that

$$
\begin{aligned}
\operatorname{size}(S) & =\operatorname{size}\left(S^{\prime}\right)-e, \\
\operatorname{size}(W) & =\operatorname{size}\left(W^{\prime}\right)-e .
\end{aligned}
$$

Corollary 4.4 (Bracket).

$$
D_{i} \circ U_{j}-U_{j} \circ D_{i}=D_{i-1} \circ U_{j-1} .
$$

Proof.

$$
D_{i} \circ U_{j}=\sum_{e \geq 0} U_{j-e} \circ D_{i-e}=U_{j} \circ D_{i}+\sum_{e \geq 1} U_{j-e} \circ D_{i-e}=U_{j} \circ D_{i}+D_{i-1} \circ U_{i-1} .
$$

4.3. $D_{i}$ stabilizes $\mathbb{B}$. We use the commutation relation of the previous section to prove that $D_{i}(\mathbb{B}) \subseteq \mathbb{B}$. First we determine the image of $\mathbf{h}_{r}$ under $D_{i}$.

Lemma 4.5. For $r \leq k$ and all $i$,

$$
D_{i}\left(\mathbf{h}_{r}\right)=\mathbf{h}_{r-i} .
$$

Proof. If $i>r$, then $D_{i}\left(\mathbf{h}_{r}\right)=0$ since there are no strong strips of size $i$ beginning at elements $w$ of length $r$. Also, by definition, $\mathbf{h}_{r-i}=0$. So suppose that $i \leq r$. Proceed by induction on $i$. If $i=1$, then

$$
D_{1}\left(\mathbf{h}_{r}\right)=\left(D_{1} \circ U_{r}\right)\left(1_{\mathbb{A}}\right)=\left(U_{r} \circ D_{1}\right)\left(1_{\mathbb{A}}\right)+\left(D_{0} \circ U_{r-1}\right)\left(1_{\mathbb{A}}\right)=0_{\mathbb{A}}+\mathbf{h}_{r-1} .
$$

Suppose the result holds for $i-1$. Then

$$
\begin{aligned}
D_{i}\left(\mathbf{h}_{r}\right) & =\left(D_{i} \circ U_{r}\right)\left(1_{\mathbb{A}}\right)=\left(U_{r} \circ D_{i}\right)\left(1_{\mathbb{A}}\right)+\left(D_{i-1} \circ U_{r-1}\right)\left(1_{\mathbb{A}}\right) \\
& =0_{\mathbb{A}}+D_{i-1}\left(\mathbf{h}_{r-1}\right)=\mathbf{h}_{r-i} .
\end{aligned}
$$

Theorem 4.6. Let $J$ be a composition. Then $D_{J}$ stabilizes $\mathbb{B}$; that is,

$$
D_{J}(\mathbb{B}) \subseteq \mathbb{B} .
$$

Proof. It suffices to prove this for the operators $D_{i}$ since $D_{J}$ is a linear combination of compositions of these operators. Since $\mathbb{B}$ is spanned by the products $\mathbf{h}_{j_{1}} \mathbf{h}_{j_{2}} \cdots \mathbf{h}_{j_{l}}$, it suffices to show that $D_{i}\left(\mathbf{h}_{j_{1}} \mathbf{h}_{j_{2}} \cdots \mathbf{h}_{j_{l}}\right) \in \mathbb{B}$. Proceed by induction on $i$ and $l$. If $l=1$, then by Lemma 4.5, $D_{i}\left(\mathbf{h}_{j_{1}}\right)=\mathbf{h}_{j_{1}-i} \in \mathbb{B}$. For $i=1$ this was proved in BSS12, Theorem 3.9]. If $l>1$, then

$$
\begin{aligned}
D_{i}\left(\mathbf{h}_{j_{1}} \mathbf{h}_{j_{2}} \cdots \mathbf{h}_{j_{l}}\right) & =\left(D_{i} \circ U_{j_{1}}\right)\left(\mathbf{h}_{j_{2}} \cdots \mathbf{h}_{j_{l}}\right) \\
& =\left(U_{j_{1}} \circ D_{i}\right)\left(\mathbf{h}_{j_{2}} \cdots \mathbf{h}_{j_{l}}\right)+\left(D_{i-1} \circ U_{j_{1}-1}\right)\left(\mathbf{h}_{j_{2}} \cdots \mathbf{h}_{j_{l}}\right) \\
& =\mathbf{h}_{j_{1}} D_{i}\left(\mathbf{h}_{j_{2}} \cdots \mathbf{h}_{j_{l}}\right)+D_{i-1}\left(\mathbf{h}_{j_{1}-1} \mathbf{h}_{j_{2}} \cdots \mathbf{h}_{j_{l}}\right) \in \mathbb{B} .
\end{aligned}
$$


Since the noncommutative $k$-Schur functions form a basis of $\mathbb{B}$, it is natural to ask for the expansion of $D_{i}\left(\mathfrak{s}_{\lambda}^{(k)}\right)$ in terms of noncommutative $k$-Schur functions. We obtain the following combinatorial description in terms of strong strips. Recall that $w_{\lambda}$ denotes the 0-Grassmannian element corresponding to the $k$-bounded partition $\lambda$ under the bijection between $\mathcal{B}^{(k)}$ and $W^{0}$.

\section{Theorem 4.7.}

$$
D_{i}\left(\mathfrak{s}_{\lambda}^{(k)}\right)=\sum_{\operatorname{size}\left(w_{\lambda} \rightarrow w_{\mu}\right)=i} \mathfrak{s}_{\mu}^{(k)} .
$$

Proof. Since $D_{i}\left(\mathfrak{s}_{\lambda}^{(k)}\right) \in \mathbb{B}$, to compute its expansion in terms of $k$-Schur functions, it suffices to compute the coefficient of $\mathbf{u}_{w}$ for 0-Grassmannian elements $w$ (see \$2.7). This is the number of strong strips $v \rightarrow w$ of length $i$ with $\mathbf{u}_{v}$ appearing as a term in $\mathfrak{s}_{\lambda}^{(k)}$. But a strong strip that ends at a 0-Grassmannian element necessarily begins at a 0-Grassmannian element [LLMS10, Proposition 2.6], and there is a unique term $\mathbf{u}_{v}$ appearing in $\mathfrak{s}_{\lambda}^{(k)}$ with $v$ a 0-Grassmannian element, namely $\mathbf{u}_{w_{\lambda}}$.

4.4. Restriction to $\mathbb{B}$. We prove that $D_{J}$ is determined by its restriction to $\mathbb{B}$ and we identify this restriction as a linear operator adjoint to multiplication by a symmetric function with respect to the pairing on $\Lambda_{(k)} \times \Lambda^{(k)}$.

Theorem 4.8. Suppose $w \in W$ and $v \in W_{0}$. Then

$$
\begin{aligned}
& U_{j}\left(\mathbf{u}_{w} \mathbf{u}_{v}\right)=U_{j}\left(\mathbf{u}_{w}\right) \mathbf{u}_{v}, \\
& D_{i}\left(\mathbf{u}_{w} \mathbf{u}_{v}\right)=D_{i}\left(\mathbf{u}_{w}\right) \mathbf{u}_{v} .
\end{aligned}
$$

Consequently, $U_{j}$ and $D_{i}$ are completely determined by their restriction to $\mathbb{B}$.

Proof. Since $U_{j}$ is left-multiplication by $\mathbf{h}_{j}$, associativity implies that $U_{j}\left(\mathbf{u}_{w} \mathbf{u}_{v}\right)=$ $U_{j}\left(\mathbf{u}_{w}\right) \mathbf{u}_{v}$, establishing the first equality. By Corollary 4.2, it suffices to show that $D_{i}\left(\mathbf{h}_{j_{1}} \mathbf{h}_{j_{2}} \cdots \mathbf{h}_{j_{l}} \mathbf{u}_{v}\right)=D_{i}\left(\mathbf{h}_{j_{1}} \mathbf{h}_{j_{2}} \cdots \mathbf{h}_{j_{l}}\right) \mathbf{u}_{v}$. Proceed by induction. The case $i=1$ was proved in [BSS12, Theorem 3.10]. Suppose the result holds for $D_{i-1}$. We prove the result also holds for $D_{i}$ by induction on $l$. If $l=1$, then

$$
D_{i}\left(\mathbf{h}_{j_{1}} \mathbf{u}_{v}\right)=\left(D_{i} \circ U_{j_{1}}\right)\left(\mathbf{u}_{v}\right)=\left(U_{j_{1}} \circ D_{i}\right)\left(\mathbf{u}_{v}\right)+\left(D_{i-1} \circ U_{j_{1}-1}\right)\left(\mathbf{u}_{v}\right) .
$$

Note that $D_{i}\left(\mathbf{u}_{v}\right)=0$ because there is no strong strip starting from $v \in W_{0}$. Also, since the result holds for $D_{i-1}$, we have

$$
\left(D_{i-1} \circ U_{j_{1}-1}\right)\left(\mathbf{u}_{v}\right)=D_{i-1}\left(\mathbf{h}_{j_{1}-1} \mathbf{u}_{v}\right)=D_{i-1}\left(\mathbf{h}_{j_{1}-1}\right) \mathbf{u}_{v}=D_{i}\left(\mathbf{h}_{j_{1}}\right) \mathbf{u}_{v} .
$$

For $l>1$, use the identity $D_{i} \circ U_{j_{1}}=U_{j_{1}} \circ D_{i}+D_{i-1} \circ U_{j_{1}-1}$ to write

$$
D_{i}\left(\mathbf{h}_{j_{1}} \mathbf{h}_{j_{2}} \cdots \mathbf{h}_{j_{l}} \mathbf{u}_{v}\right)=\left(U_{j_{1}} \circ D_{i}\right)\left(\mathbf{h}_{j_{2}} \cdots \mathbf{h}_{j_{l}} \mathbf{u}_{v}\right)+D_{i-1}\left(\mathbf{h}_{j_{1}-1} \mathbf{h}_{j_{2}} \cdots \mathbf{h}_{j_{l}} \mathbf{u}_{v}\right) .
$$

Since the product $\mathbf{h}_{j_{2}} \cdots \mathbf{h}_{j_{l}}$ involves less than $l$ terms, by induction we have that

$$
\left(U_{j_{1}} \circ D_{i}\right)\left(\mathbf{h}_{j_{2}} \cdots \mathbf{h}_{j_{l}} \mathbf{u}_{v}\right)=U_{j_{1}}\left(D_{i}\left(\mathbf{h}_{j_{2}} \cdots \mathbf{h}_{j_{l}}\right) \mathbf{u}_{v}\right)=\left(U_{j_{1}} \circ D_{i}\right)\left(\mathbf{h}_{j_{2}} \cdots \mathbf{h}_{j_{l}}\right) \mathbf{u}_{v} .
$$

Since the result holds for $D_{i-1}$, we have that

$$
D_{i-1}\left(\mathbf{h}_{j_{1}-1} \mathbf{h}_{j_{2}} \cdots \mathbf{h}_{j_{l}} \mathbf{u}_{v}\right)=D_{i-1}\left(\mathbf{h}_{j_{1}-1} \mathbf{h}_{j_{2}} \cdots \mathbf{h}_{j_{l}}\right) \mathbf{u}_{v} .
$$

Hence, $D_{i}\left(\mathbf{h}_{j_{1}} \mathbf{h}_{j_{2}} \cdots \mathbf{h}_{j_{l}} \mathbf{u}_{v}\right)=D_{i}\left(\mathbf{h}_{j_{1}} \mathbf{h}_{j_{2}} \cdots \mathbf{h}_{j_{l}}\right) \mathbf{u}_{v}$, as desired. 
We next identify the restriction of $D_{J}$ to $\mathbb{B}$. For a composition $J$, let $s_{J}$ denote the ribbon Schur function indexed by $J$ (for a good introduction to ribbon Schur functions, see for instance [BTvW06]) and let $\overline{s_{J}}$ denote its image in $\Lambda^{(k)}$. Recall that $\overline{s_{J}} \perp: \Lambda_{(k)} \rightarrow \Lambda_{(k)}$ is the linear operator adjoint to multiplication by $\overline{s_{J}}$ in $\Lambda^{(k)}$. We also denote the corresponding linear operator on $\mathbb{B}$ by $\overline{s_{J}} \perp$.

Theorem 4.9. The restriction of $D_{J}$ to $\mathbb{B}$ is $\overline{s_{J}}{ }^{\perp}$. Consequently, $D_{J}$ is the extension to $\mathbb{A}$, as defined in 4.1 , of $\overline{s_{J}} \perp: \mathbb{B} \rightarrow \mathbb{B}$.

Proof. In the following, let $D_{J}\left(s_{\lambda}^{(k)}\right)$ denote the image $D_{J}\left(\mathfrak{s}_{\lambda}^{(k)}\right)$ under the isomorphism $\mathbb{B} \rightarrow \Lambda_{(k)}$. We will prove, for all $s_{\lambda}^{(k)}$ and $\widetilde{F}_{\mu}$,

$$
\left\langle D_{J}\left(s_{\lambda}^{(k)}\right), \widetilde{F}_{\mu}\right\rangle=\left\langle s_{\lambda}^{(k)}, \overline{s_{J}} \widetilde{F}_{\mu}\right\rangle .
$$

Proceed by induction on the length of $J=\left[j_{1}, j_{2}, \ldots, j_{l}\right]$. Suppose $l=1$. Then it suffices to prove that

$$
\left\langle D_{j}\left(s_{\lambda}^{(k)}\right), \widetilde{F}_{\mu}\right\rangle=\left\langle s_{\lambda}^{(k)}, \overline{h_{j}} \widetilde{F}_{\mu}\right\rangle .
$$

But this follows immediately from Theorem 4.7 and the Pieri rule: $\overline{h_{j}} \widetilde{F}_{w_{\mu}}=\sum \widetilde{F}_{w_{\lambda}}$ with the sum running over all strong strips $w_{\lambda} \rightarrow w_{\mu}$ of size $j$ (see LLMS10, Theorem 4.13]).

Now suppose the result holds for compositions of length less than $l$. Let $J=$ $\left[j_{1}, j_{2}, \ldots, j_{l}\right]$. Observe that

$$
D_{J}=D_{j_{1}} \circ D_{\left[j_{2}, \ldots, j_{l}\right]}-D_{\left[j_{1}+j_{2}, \ldots, j_{l}\right]},
$$

so by induction and the product rule for ribbon Schur functions [Mac, §169],

$$
\begin{aligned}
D_{J} & ={\overline{s_{j_{1}}}}^{\perp} \circ \overline{s_{\left[j_{2}, \ldots, j_{l}\right]}}{ }^{\perp}-\overline{s_{\left[j_{1}+j_{2}, \ldots, j_{l}\right]}} \perp \\
& =\overline{s_{j_{1}} s_{\left[j_{2}, \ldots, j_{l}\right]}-s_{\left[j_{1}+j_{2}, \ldots, j_{l}\right]}} \perp=\overline{s_{J}} \perp .
\end{aligned}
$$

Theorem 4.10. The operators $D_{J}$ and $D_{K}$ commute.

Proof. $\mathbb{A}$ is spanned by elements of the form $\mathbf{b u}_{w}$ with $\mathbf{b} \in \mathbb{B}$ and $w \in W_{0}$ (Proposition 4.1), so it suffices to prove this for these elements. Combining Theorems 4.8 and 4.9, we have

$$
\begin{aligned}
& \left(D_{K} \circ D_{J}\right)\left(\mathbf{b u}_{w}\right)=\left(D_{K} \circ D_{J}\right)(\mathbf{b}) \mathbf{u}_{w}=\left({\overline{s_{K}}}^{\perp} \circ{\overline{s_{J}}}^{\perp}\right)(\mathbf{b}) \mathbf{u}_{w} \\
& =\left({\overline{s_{J}}}^{\perp} \circ{\overline{s_{K}}}^{\perp}\right)(\mathbf{b}) \mathbf{u}_{w}=\left(D_{J} \circ D_{K}\right)(\mathbf{b}) \mathbf{u}_{w}=\left(D_{J} \circ D_{K}\right)\left(\mathbf{b} \mathbf{u}_{w}\right),
\end{aligned}
$$

where the third equality comes from the commutation of symmetric functions.

Remark 4.11. Theorem 4.10 implies that there is a bijection between strong strips $w \rightarrow u$ with ascent composition $I \boxplus J$ or $I \bullet J$ and strong strips $w \rightarrow u$ with ascent composition $J \boxplus I$ or $J \boxminus I$. We do not know of an explicit bijection.

\section{Strong Schur FUnCtions}

Lam, Lapointe, Morse, and Shimozono LLMS10 generalized the $k$-Schur functions to a larger set of functions called the strong Schur functions. We use the properties of the operators developed in the previous section to prove a series of their conjectures [LLMS10, Conjecture 4.18] regarding these functions. 
5.1. Strong Schur functions are symmetric functions. For $u, v \in W$, define the strong Schur function

$$
\operatorname{Strong}_{u / v}=\sum_{u \rightarrow \cdots \rightarrow v \in \mathcal{G} \downarrow} L_{\operatorname{ascomp}(u \rightarrow \cdots \rightarrow v)},
$$

where $L_{J}$ denotes the fundamental quasi-symmetric function indexed by the composition $J$. In [LLMS10, it was shown that Strong id $_{u}$ is a symmetric function and that when $u$ is 0 -Grassmannian, it is a $k$-Schur function.

Remark 5.1. The definition given here is a reformulation of that in [LLMS10. They defined Strong $_{u / v}$ as the generating function of "strong tableaux"; the above definition is obtained from theirs by lumping together tableaux of the same "weight", yielding the expansion in terms of monomial quasi-symmetric functions below.

Theorem 5.2 ([LLMS10, Conjecture 4.18(1)]). Strong ${ }_{u / v}$ is a symmetric function. Furthermore, it expands positively in the monomial basis $m_{\lambda}$ of $\Lambda$ :

$$
\operatorname{Strong}_{u / v}=\sum_{\lambda}\left\langle D^{\lambda}\left(\mathbf{u}_{u}\right), \mathbf{u}_{v}\right\rangle_{\mathbb{A}} m_{\lambda}
$$

where $D^{\lambda}=D_{\lambda_{1}} \circ \cdots \circ D_{\lambda_{l}}$.

Proof. The coefficient of the fundamental quasi-symmetric function $L_{J}$ in Strong $u / v$ is the number of paths in $\mathcal{G}^{\downarrow}$ from $u$ to $v$ with ascent composition equal to $J$. This is precisely the coefficient of $\mathbf{u}_{v}$ in $D_{J}\left(\mathbf{u}_{u}\right)$. Hence,

$$
\operatorname{Strong}_{u / v}=\sum_{J \models \ell(u)-\ell(v)}\left\langle D_{J}\left(\mathbf{u}_{u}\right), \mathbf{u}_{v}\right\rangle_{\mathbb{A}} L_{J} .
$$

Recall that $L_{J}=\sum_{I \succeq J} M_{I}$, where $M_{I}$ denotes the monomial quasi-symmetric function indexed by the composition $I=\left[i_{1}, \ldots, i_{r}\right]$. Thus,

$$
\begin{aligned}
\operatorname{Strong}_{u / v} & =\sum_{J}\left\langle D_{J}\left(\mathbf{u}_{u}\right), \mathbf{u}_{v}\right\rangle_{\mathbb{A}} \sum_{I \succeq J} M_{I} \\
& =\sum_{I}\left(\sum_{J \preceq I}\left\langle D_{J}\left(\mathbf{u}_{u}\right), \mathbf{u}_{v}\right\rangle_{\mathbb{A}}\right) M_{I} \\
& =\sum_{I}\left\langle D^{I}\left(\mathbf{u}_{u}\right), \mathbf{u}_{v}\right\rangle_{\mathbb{A}} M_{I},
\end{aligned}
$$

where $D^{I}=D_{i_{1}} \circ \cdots \circ D_{i_{r}}$. Since the operators $D_{i}$ and $D_{j}$ commute for all $i$ and $j$, the operator $D^{I}$ depends only on the underlying partition $\lambda(I)$ of $I$. Hence,

$$
\begin{aligned}
\operatorname{Strong}_{u / v} & =\sum_{\lambda} \sum_{\lambda(I)=\lambda}\left\langle D^{I}\left(\mathbf{u}_{u}\right), \mathbf{u}_{v}\right\rangle_{\mathbb{A}} M_{I} \\
& =\sum_{\lambda}\left\langle D^{\lambda}\left(\mathbf{u}_{u}\right), \mathbf{u}_{v}\right\rangle_{\mathbb{A}} \sum_{\lambda(I)=\lambda} M_{I} \\
& =\sum_{\lambda}\left\langle D^{\lambda}\left(\mathbf{u}_{u}\right), \mathbf{u}_{v}\right\rangle_{\mathbb{A}} m_{\lambda},
\end{aligned}
$$

where $m_{\lambda}$ is the monomial symmetric function. In particular, Strong ${ }_{u / v} \in \Lambda$. 
If $u$ and $v$ are 0 -Grassmannian elements, we write $\operatorname{Strong}_{\mu / \nu}$ instead of $\operatorname{Strong}_{u / v}$, where $\mu$ and $\nu$ are the $k$-bounded partitions corresponding to $u$ and $v$, respectively. It follows from $\$ 2.7$ (as in the proof of Theorem 4.7) that the coefficient of $\mathbf{u}_{v}$ in $D^{\lambda}\left(\mathbf{u}_{u}\right)$ is the coefficient of $s_{\nu}^{(k)}$ in the expansion of $D^{\lambda}\left(s_{\mu}^{(k)}\right)$ in terms of $k$-Schur functions. Thus,

$$
\left\langle D^{\lambda}\left(\mathbf{u}_{u}\right), \mathbf{u}_{v}\right\rangle_{\mathbb{A}}=\left\langle D^{\lambda}\left(s_{\mu}^{(k)}\right), \widetilde{F}_{\nu}\right\rangle=\left\langle s_{\mu}^{(k)}, \overline{h_{\lambda}} \widetilde{F}_{\nu}\right\rangle,
$$

where the last equality follows from the fact that the restriction of $D^{\lambda}$ to $\mathbb{B}$ is the adjoint to the multiplication by $\overline{h_{\lambda}}$ (Theorem 4.9) .

Corollary 5.3. If $u$ and $v$ are 0-Grassmannian elements corresponding to the $k$ bounded partitions $\mu$ and $\nu$, respectively, then

$$
\operatorname{Strong}_{\mu / \nu}=\sum_{\lambda}\left\langle s_{\mu}^{(k)}, \overline{h_{\lambda}} \widetilde{F}_{\nu}\right\rangle m_{\lambda} .
$$

5.2. Strong Schur functions belong to $\Lambda_{(k)}$. Next we verify the second part of Conjecture 4.18 from [LLMS10]. Recall that for a linear operator $f$ on $\mathbb{B}$, we denote by $\widehat{f}$ its extension to $\mathbb{A}$ as defined in 4.1 .

Theorem 5.4. Let $u, v \in W$. The strong Schur function Strong ${ }_{u / v}$ lies in $\Lambda_{(k)}$. Furthermore, we have the expansion in homogeneous symmetric functions:

$$
\operatorname{Strong}_{u / v}=\sum_{\lambda \in \mathcal{B}^{(k)}}\left\langle\widehat{m_{\lambda}^{\perp}}\left(\mathbf{u}_{u}\right), \mathbf{u}_{v}\right\rangle_{\mathbb{A}} h_{\lambda} .
$$

Proof. Since the $m_{\mu}$ form a basis of $\Lambda$, there exist coefficients $\mathfrak{c}_{\lambda, \mu}$ for which $h_{\lambda}=$ $\sum_{\mu} \mathfrak{c}_{\lambda, \mu} m_{\mu}$. Hence,

$$
\begin{aligned}
\operatorname{Strong}_{u / v} & =\sum_{\lambda}\left\langle D^{\lambda}\left(\mathbf{u}_{u}\right), \mathbf{u}_{v}\right\rangle_{\mathbb{A}} m_{\lambda} \\
& =\sum_{\lambda}\left\langle\widehat{h_{\lambda}^{\perp}}\left(\mathbf{u}_{u}\right), \mathbf{u}_{v}\right\rangle_{\mathbb{A}} m_{\lambda} \\
& =\sum_{\lambda, \mu}\left\langle\mathfrak{c}_{\lambda, \mu} \widehat{m_{\mu}^{\perp}}\left(\mathbf{u}_{u}\right), \mathbf{u}_{v}\right\rangle_{\mathbb{A}} m_{\lambda} \\
& =\sum_{\mu}\left\langle\widehat{m_{\mu}^{\perp}}\left(\mathbf{u}_{u}\right), \mathbf{u}_{v}\right\rangle_{\mathbb{A}} \sum_{\lambda} \mathfrak{c}_{\lambda, \mu} m_{\lambda} \\
& =\sum_{\mu}\left\langle\widehat{m_{\mu}^{\perp}}\left(\mathbf{u}_{u}\right), \mathbf{u}_{v}\right\rangle_{\mathbb{A}} h_{\mu} .
\end{aligned}
$$

Since $m_{\mu}^{\perp}=0$ for any partition $\mu$ that is not $k$-bounded, the above summation runs over $k$-bounded partitions.

5.3. Expansions of strong Schur functions. Since Strong ${ }_{u / v}$ lies in $\Lambda_{(k)}$, it has an expansion in terms of $k$-Schur functions. The third part of Conjecture 4.18 of [LLMS10] deals with the coefficients in this expansion.

Theorem 5.5. Let $u, v \in W$. Then

$$
\operatorname{Strong}_{u / v}=\sum_{\lambda \in \mathcal{B}^{(k)}}\left\langle\widehat{\widetilde{F}_{\lambda}^{\perp}}\left(\mathbf{u}_{u}\right), \mathbf{u}_{v}\right\rangle_{\mathbb{A}} s_{\lambda}^{(k)} .
$$


Proof. By using the expansions $h_{\lambda}=\sum_{\tau} K_{\tau, \lambda}^{(k)} s_{\tau}^{(k)}$ and $\widetilde{F}_{\tau}=\sum_{\lambda} K_{\tau, \lambda}^{(k)} m_{\lambda}$,

$$
\begin{aligned}
\operatorname{Strong}_{u / v} & =\sum_{\lambda \in \mathcal{B}^{(k)}}\left\langle\widehat{m_{\lambda}^{\perp}}\left(\mathbf{u}_{u}\right), \mathbf{u}_{v}\right\rangle_{\mathbb{A}} h_{\lambda} \\
& =\sum_{\lambda \in \mathcal{B}^{(k)}}\left\langle\widehat{m_{\lambda}^{\perp}}\left(\mathbf{u}_{u}\right), \mathbf{u}_{v}\right\rangle_{\mathbb{A}} \sum_{\tau \in \mathcal{B}^{(k)}} K_{\tau, \lambda}^{(k)} s_{\tau}^{(k)} \\
& =\sum_{\tau \in \mathcal{B}^{(k)}}\left\langle\sum_{\lambda \in \mathcal{B}^{(k)}} K_{\tau, \lambda}^{(k)} \widehat{m_{\lambda}^{\perp}}\left(\mathbf{u}_{u}\right), \mathbf{u}_{v}\right\rangle_{\mathbb{A}} s_{\tau}^{(k)} \\
& =\sum_{\tau \in \mathcal{B}^{(k)}}\left\langle\widehat{\widetilde{F}_{\tau}^{\perp}}\left(\mathbf{u}_{u}\right), \mathbf{u}_{v}\right\rangle_{\mathbb{A}} s_{\tau}^{(k)} .
\end{aligned}
$$

The third part of Conjecture 4.18 of LLMS10 states that the coefficients in the above summation are nonnegative; more explicitly, that they are the structure constants of the Schubert classes for the cohomology of the affine flag variety. We prove this conjecture for 0-Grassmannian elements; the general case is still open.

Corollary 5.6 (LLMS10, Conjecture 4.18(3)]). Let $\mu$ and $\nu$ be $k$-bounded partitions. The coefficient of $s_{\lambda}^{(k)}$ in Strong $_{\mu / \nu}$ is the coefficient of $\widetilde{F}_{\mu}$ in $\widetilde{F}_{\lambda} \widetilde{F}_{\nu}$ :

$$
\operatorname{Strong}_{\mu / \nu}=\sum_{\lambda \in \mathcal{B}^{(k)}}\left\langle s_{\mu}^{(k)}, \widetilde{F}_{\lambda} \widetilde{F}_{\nu}\right\rangle s_{\lambda}^{(k)} .
$$

In particular, the strong Schur functions Strong $_{\mu / \nu}$ are $k$-Schur positive.

Proof. If $u$ and $v$ are 0 -Grassmannian, with $u=w_{\mu}$ and $v=w_{\lambda}$, then the coefficient in Theorem 5.5 reduces to

$$
\left\langle\widehat{\widetilde{F}_{\lambda}^{\perp}}\left(\mathbf{u}_{u}\right), \mathbf{u}_{v}\right\rangle_{\mathbb{A}}=\left\langle\widetilde{F}_{\lambda}^{\perp}\left(s_{\mu}^{(k)}\right), \widetilde{F}_{\nu}\right\rangle=\left\langle s_{\mu}^{(k)}, \widetilde{F}_{\lambda} \widetilde{F}_{\nu}\right\rangle .
$$

These coefficients are known to be nonnegative because they are the structure constants of the Schubert classes in the cohomology of the affine Grassmannian Lam08.

Corollary 5.7. Let $\mu$ and $\nu$ be $k$-bounded partitions. Then the skew $k$-Schur function is

$$
s_{\mu / \nu}^{(k)}:=\widetilde{F}_{\nu}^{\perp}\left(s_{\mu}^{(k)}\right)=\operatorname{Strong}_{\mu / \nu} .
$$

Proof. By Corollary [5.6, we have Strong ${ }_{\mu}=s_{\mu}^{(k)}$. Thus, the coefficient of $s_{\lambda}^{(k)}$ in the left-hand side is $\left\langle\widetilde{F}_{\nu}^{\perp}\left(s_{\mu}^{(k)}\right), \widetilde{F}_{\lambda}\right\rangle=\left\langle s_{\mu}^{(k)}, \widetilde{F}_{\nu} \widetilde{F}_{\lambda}\right\rangle$, which is the coefficient of $s_{\lambda}^{(k)}$ in Strong $_{\mu / \nu}$.

Consequently, we obtain an explicit combinatorial description of the skew $k$ Schur function $s_{\lambda / \mu}^{(k)}$ since the strong Schur function Strong ${ }_{\mu / \nu}$ has an explicit combinatorial description in terms of "strong tableaux" (see [LLMS10] for details). 


\section{ACKNOWLEDGEMENTS}

We would like to thank Nantel Bergeron, Sergey Fomin, Thomas Lam, Jennifer Morse, Anne Schilling, and Mike Zabrocki for helpful discussions.

This research was facilitated by computer exploration using the open-source mathematical software system Sage [Ste82] and its algebraic combinatorics features developed by the Sage-Combinat community SCc12.

We are very grateful to the anonymous referee for useful comments that improved this article.

\section{REFERENCES}

[BSS12] Chris Berg, Franco Saliola, and Luis Serrano, The down operator and expansions of near rectangular $k$-Schur functions, 24th International Conference on Formal Power Series and Algebraic Combinatorics (FPSAC 2012), Discrete Math. Theor. Comput. Sci. Proc., AR, Assoc. Discrete Math. Theor. Comput. Sci., Nancy, 2012, pp. 433-444 (English, with English and French summaries). MR.2958018

[BMSvW00] Nantel Bergeron, Stefan Mykytiuk, Frank Sottile, and Stephanie van Willigenburg, Noncommutative Pieri operators on posets, J. Combin. Theory Ser. A 91 (2000), no. 1-2, 84-110, DOI 10.1006/jcta.2000.3090. In memory of Gian-Carlo Rota. MR1779776 (2002d:05122)

[BTvW06] Louis J. Billera, Hugh Thomas, and Stephanie van Willigenburg, Decomposable compositions, symmetric quasisymmetric functions and equality of ribbon Schur functions, Adv. Math. 204 (2006), no. 1, 204-240, DOI 10.1016/j.aim.2005.05.014. MR2233132 (2007b:05207)

[Lam10] T. Lam, Stanley symmetric functions and Peterson algebras (2010). ArXiv e-prints.

[Lam06] Thomas Lam, Affine Stanley symmetric functions, Amer. J. Math. 128 (2006), no. 6, 1553-1586. MR 2275911 (2008b:05178)

[Lam08] Thomas Lam, Schubert polynomials for the affine Grassmannian, J. Amer. Math. Soc. 21 (2008), no. 1, 259-281, DOI 10.1090/S0894-0347-06-00553-4. MR2350056 (2009a:05207)

[LLMS10] Thomas Lam, Luc Lapointe, Jennifer Morse, and Mark Shimozono, Affine insertion and Pieri rules for the affine Grassmannian, Mem. Amer. Math. Soc. 208 (2010), no. 977, xii+82, DOI 10.1090/S0065-9266-10-00576-4. MR2741963 (2012f:05314)

[LS10] Thomas Lam and Mark Shimozono, Quantum cohomology of G/P and homology of affine Grassmannian, Acta Math. 204 (2010), no. 1, 49-90, DOI 10.1007/s11511010-0045-8. MR2600433(2011h:14082)

[LS07] Thomas F. Lam and Mark Shimozono, Dual graded graphs for Kac-Moody algebras, Algebra Number Theory 1 (2007), no. 4, 451-488, DOI 10.2140/ant.2007.1.451. MR2368957 (2009a:05209)

[LLM03] L. Lapointe, A. Lascoux, and J. Morse, Tableau atoms and a new Macdonald positivity conjecture, Duke Math. J. 116 (2003), no. 1, 103-146, DOI 10.1215/S00127094-03-11614-2. MR 1950481(2004c:05208)

[LM03] L. Lapointe and J. Morse, Schur function analogs for a filtration of the symmetric function space, J. Combin. Theory Ser. A 101 (2003), no. 2, 191-224, DOI 10.1016/S0097-3165(02)00012-2. MR.1961543(2004c:05209)

[LM05] Luc Lapointe and Jennifer Morse, Tableaux on $k+1$-cores, reduced words for affine permutations, and $k$-Schur expansions, J. Combin. Theory Ser. A 112 (2005), no. 1, 44-81, DOI 10.1016/j.jcta.2005.01.003. MR2167475 (2006j:05214)

[LM07] Luc Lapointe and Jennifer Morse, A $k$-tableau characterization of $k$-Schur functions, Adv. Math. 213 (2007), no. 1, 183-204, DOI 10.1016/j.aim.2006.12.005. MR2331242 (2008c:05187)

[LM08] Luc Lapointe and Jennifer Morse, Quantum cohomology and the $k$-Schur basis, Trans. Amer. Math. Soc. 360 (2008), no. 4, 2021-2040, DOI 10.1090/S0002-994707-04287-0. MR2366973(2009d:14072) 
[LL12] Naichung Conan Leung and Changzheng Li, Gromov-Witten invariants for $G / B$ and Pontryagin product for $\Omega K$, Trans. Amer. Math. Soc. 364 (2012), no. 5, 2567-2599, DOI 10.1090/S0002-9947-2012-05438-9. MR2888220 (2012m:14107)

[Mac] P.A. MacMahon, Combinatory analysis, Combinatory Analysis, no. v. 2. The University Press, 1916.

[SCc12] The Sage-Combinat community, Sage-Combinat: enhancing sage as a toolbox for computer exploration in algebraic combinatorics, The Sage Development Team (2012).

[Sta] Richard P. Stanley, Enumerative combinatorics. Vol. 2, Cambridge Studies in Advanced Mathematics, vol. 62. Cambridge University Press, Cambridge, 1999. With a foreword by Gian-Carlo Rota and appendix 1 by Sergey Fomin. MR 1676282 (2000k:05026)

[Ste82] E. M. Stein, The development of square functions in the work of A. Zygmund, Bull. Amer. Math. Soc. (N.S.) 7 (1982), no. 2, 359-376, DOI 10.1090/S0273-0979-198215040-6. MR663787 (83i:42001)

Department of Mathematics, University of Quebec at Montreal, Montreal, Quebec, CANADA H8C $3 \mathrm{P} 8$

Department of Mathematics, University of Quebec at Montreal, Montreal, Quebec, CANADA H8C $3 \mathrm{P} 8$

Department of Mathematics, University of Quebec at Montreal, Montreal, Quebec, CANADA H8C $3 \mathrm{P} 8$ 